Julia Modrejewski, Johanna-Gabriela Walter*, Imme Kretschmer, Evren Kemal, Mark Green, Hamza Belhadj, Cornelia Blume and Thomas Scheper

\title{
Aptamer-modified polymer nanoparticles for targeted drug delivery
}

DOI 10.1515/bnm-2015-0027

Received December 30, 2015; accepted March 15, 2016; previously published online April 27, 2016

\begin{abstract}
The purpose of this study was to develop a model system for targeted drug delivery. This system should enable targeted drug release at a certain tissue in the body. In conventional drug delivery systems, drugs are often delivered unspecifically resulting in unwarranted adverse effects. To circumvent this problem, there is an increasing demand for the development of intelligent drug delivery systems allowing a tissue-specific mode of delivery. Within this study, nanoparticles consisting of two biocompatible polymers are used. Because of their small size, nanoparticles are well-suited for effective drug delivery. The small size affects their movement through cell and tissue barriers. Their cellular uptake is easier when compared to larger drug delivery systems. Paclitaxel was encapsulated into the nanoparticles as a model drug, and to achieve specific targeting an aptamer directed against lung cancer cells was coupled to the nanoparticles surface. Nanoparticles were characterized by dynamic light scattering (DLS), transmission electron microscopy (TEM), fourier transform infrared spectroscopy (FTIR), and nanotracking analysis (NTA). Also their surface charge was characterized from $\zeta$-potential measurements. Their preparation was optimized and subsequently specificity of drugloaded and aptamer-functionalized nanoparticles was investigated using lung cancer cells.
\end{abstract}

Keywords: aptamer; HOOC-PEG-PCL; mPEG-PCL; nanoparticles.

*Corresponding author: Johanna-Gabriela Walter, Institut für Technische Chemie, Leibniz Universität Hannover, Callinstrasse 5, 30167 Hannover, Germany, Phone: +49-511-762-2968, E-mail:walter@iftc.uni-hannover.de Julia Modrejewski, Imme Kretschmer, Evren Kemal, Mark Green, Hamza Belhadj, Cornelia Blume and Thomas Scheper: Institut für Technische Chemie, Leibniz Universität Hannover, Hannover, Germany

\section{Introduction}

Today many therapeutics have poor pharmacokinetic and biopharmaceutical properties which hamper their application in long-term therapy [1-4]. This is due to the systemic application of those drugs. Especially chemotherapeutics exert consequently unspecific toxic side effects on non-target tissues [5]. To solve these problems, many drug delivery systems like micelles, vesicles or nanospheres are available $[4,6]$. These systems are designed for transporting a pharmaceutical compound in the body (Jain PharmaBiotech, Basel, 2008). Currently, they have several limitations like non-specific drug delivery [7]. Furthermore, most of these systems cannot penetrate the cell membrane to induce cytotoxicity. In this context, targeted drug delivery systems are a very promising alternative, as they allow for specific delivery as well as efficient intracellularization.

Targeted drug delivery is a preferred method for drug administration that increases the concentration of the drug at the desired sites of action, while leaving other tissues of the body unaffected [4]. This kind of release is one special focus of nanomedicine $[4,8]$. Because of their small sizes, nanoparticles are able to penetrate inflammatory sites, tumors or microcapillaries [9]. They are also able to cross the blood-brain barrier unlike other drug delivery systems with bigger sizes [9]. The small size also allows a high cellular uptake by a variety of cell types [9, 10]. Regarding drug release nanosized drug carrier have a larger surface area-to-volume ratio meaning that they have a greater efficiency regarding drug delivery $[9,11]$.

In one common design of drug delivery systems, nanoparticles are loaded with drugs and specificity is achieved by conjugation with a ligand that solely binds to diseased cells. Interactions with healthy tissue should be hampered [12]. Consequently, the advantages of a targeted drug delivery system are reduction of adverse effects in combination with increased efficacy [13, 14]. In conventional drug delivery systems lacking specific ligands, the drug diffuses in the whole body by systemic blood circulation without any specific mechanism facilitating accumulation at the desired site of action. Under most 
circumstances only a small proportion of therapeutics reaches the target organ $[7,15]$.

A targeted drug delivery system has to be constructed in the following way: the carrier material needs to be biocompatible and biodegradable [6]. The material could be biological such as lipids, lactic acid or chitosan, or it could be chemical such as titan dioxide or cadmium [6]. In this study, methoxypolyethylene glycol (mPEG-PCL) and R-carboxyl polyethylene glycol (HOOC-PEG-PCL) were used, which are FDA approved biocompatible polymers. PEG-PCL is nontoxic and an amphiphilic copolymer [16, 17]. PEG polymers have a high colloidal stability and a resistance against protein adsorption so non-specific nanoparticle interactions with cell membranes are prevented [18]. A high stability of the used carrier material is necessary for increasing efficiency of drug by prolongation of nanoparticles residence time in the circulation [9]. For that reason, two compounds containing PEG moieties were used in this study. Nanoparticles were prepared by using solvent evaporation method [19]. Gao et al. have developed a targeted drug delivery system which consists of mPEG-PCL, HOOC-PEG-PCL and maleimide-PEG-PCL (MAL-PEG-PCL) and is functionalized with an aptamer (AS11411) and a 12-amino acid peptide. The AS111411 aptamer binds to necleolin, which is a highly expressed protein in the plasma membrane of cancer cells like glioma cells. The TGN peptide is selected by an in vivo phage display screening and allows modified nanoparticles the easier penetration of the blood brain barrier compared with the native phage [19]. In this study we are transferring this system to two other aptamers which are specific for lung cancer cells and load the drug delivery system with the anticancer drug paclitaxel.

The drug can either be encapsulated in the hydrophobic core of the carrier or coupled to its surface depending on the drugs' lipophilicity [9]. In this study, paclitaxel (PTX/Taxol) was used. PTX was originally isolated from pacific yew bark and belongs to the group of taxanes. It exhibits an anti-neoplastic activity against various types of tumors [16] qualifying PTX for the therapy of lung cancer. PTX operates as inhibitor of cell mitosis by binding to $\beta$-tubulin and disturbing the degradation of microtubules [20].

In order to achieve specific delivery at the desired site of action, the surface of the particles has to be modified with a targeting molecule [12]. This could be an aptamer, antibody, peptide or protein [21, 22]. Based on their high affinity and specificity, aptamers can be used as targeting ligands in a broad range of applications including affinity separation, analytics and cell targeting [23]. Aptamers are short single-stranded or double-stranded RNA/
DNA molecules which are able to detect cell-specific molecules like receptors present on the cell surface with a high selectivity. In comparison to antibodies, aptamers have a high stability, a straight forward modification potential and lack of immunogenicity [24, 25]. Aptamers are recovered by a procedure named SELEX (Systematic Evolution of Ligands by Exponential Enrichment) [26]. This procedure selects those oligonucleotides which are binding the desired target with the highest affinity [26, 27]. The mentioned characteristics encouraged us to use the aptamers S15 and S6 which specifically bind to lung cancer cells as a ligand for the model targeted drug delivery system [25].

To evaluate the cytotoxicity of the developed system the lung cancer cell line A549 and the cervix cancer cell line HeLa were used. While A549 cells are the target cells, against which the used aptamers have been selected, a limited affinity of the aptamers has also been reported against HeLa cells. This cross-reactivity is most likely based on a molecular target that is present on the surface of both cell lines.

The aim of this study was to develop a model system for targeted drug delivery, optimizing its preparation and investigating the cytotoxicity as well as the specificity of the system. The nanoparticles were characterized by dynamic light scattering (DLS) in terms of their hydrodynamic diameter, their surface charge by measuring the $\zeta$-potential and by transmission electron microscopy (TEM) in terms of their morphology. The functionalization with aptamers was confirmed by agarose gel electrophoresis. PTX encapsulation efficiency was determined by HPLC. In order to demonstrate the applicability of the developed system in targeted drug delivery, cytotoxicity tests were performed.

\section{Results and discussion}

The aim of this study was to develop a model system for targeted drug delivery, optimizing it and investigating the cytotoxicity as well as the specificity of the system. Therefore, first corresponding copolymers mPEG-PCL and HOOC-PEG-PCL were prepared which than were used to produce the PEG-PCL nanoparticles. PTX was encapsulated during the preparation procedure and afterwards NPs were functionalized with S6 and S15 aptamers. In the following, NPs are named according to their composition: PEG-PCL-S15 (PTX) and PEG-PCL-S6 (PTX) for aptamermodified NPs containing PTX, and PEG-PCL-S15 and PEGPCL-S6 for NPs without PTX. 


\section{Preparation of copolymers}

First the copolymers were prepared by a ringopening copolymerization of $\mathrm{mPEG}$ and $\varepsilon$-caprolactone as the first compound and HOOC-PEG and $\varepsilon$-caprolactone as the second compound. The success of the copolymerization of the two compounds was determined by ${ }^{1} \mathrm{H}$ NMR. Figure 1 demonstrates the result of the copolymerization of HOOCPEG-PCL. The peak of PEG protons was at $\delta 3.7 \mathrm{ppm}$ and that one of the PCL protons at $\delta 2.3 \mathrm{ppm}$. The peak at of solvent protons is at $\delta 7.2 \mathrm{ppm}$. The results were in accordance to those reported before by Gao et al. and thus confirmed successfully [19].

Figure 2 shows the FTIR spectra of HOOC-PEGPCL and mPEG-PCL. In the spectrum of HOOC-PEG-PCL (Figure 2), the sharp and intense bands at $1724 \mathrm{~cm}^{-1}$ and $1376 \mathrm{~cm}^{-1}$ were attributed to the presence of carboxylic ester $(\mathrm{C}=0)$ and ether $(\mathrm{C}-\mathrm{O})$ groups. The same result is shown in Figure 2. The sharp and intense bands at $1727 \mathrm{~cm}^{-1}$ and $1347 \mathrm{~cm}^{-1}$ were attributed to the presence of carboxylic ester and ether groups. This indicates that the formation of both copolymers was successfully.

\section{Optimizing preparation}

Particles were prepared using the solvent evaporation method. The two compounds mPEG-PCL and HOOC-PEGPCL were dissolved in DCM and then dropwise added to a stirred sodium cholate hydrate solution. To optimize the preparation procedure, the stirring rate was modified and the resulting nanoparticles were characterized by DLS.

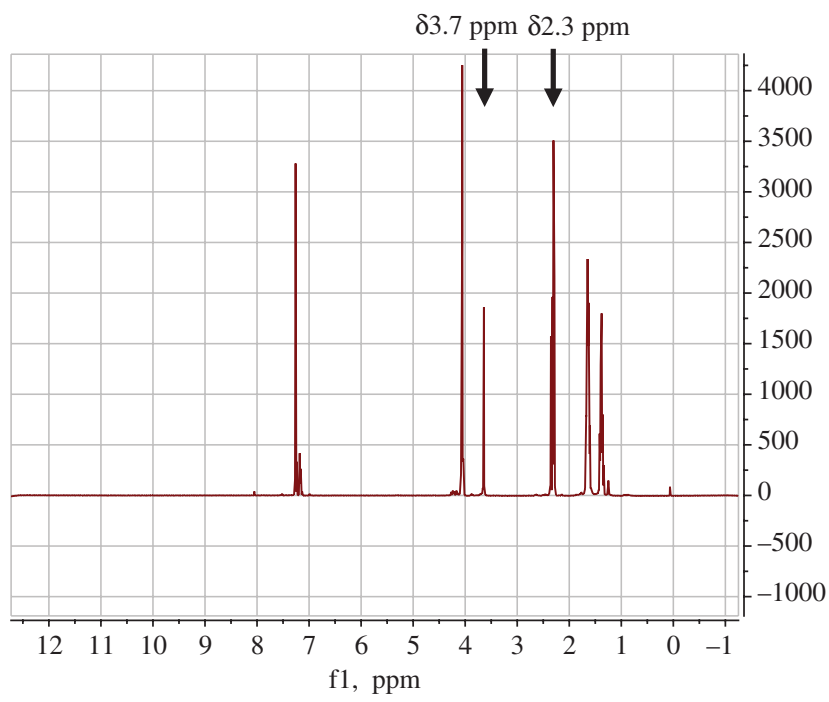

Figure 1: ${ }^{1} \mathrm{H}$ NMR o HOOC-PEG-PCL.

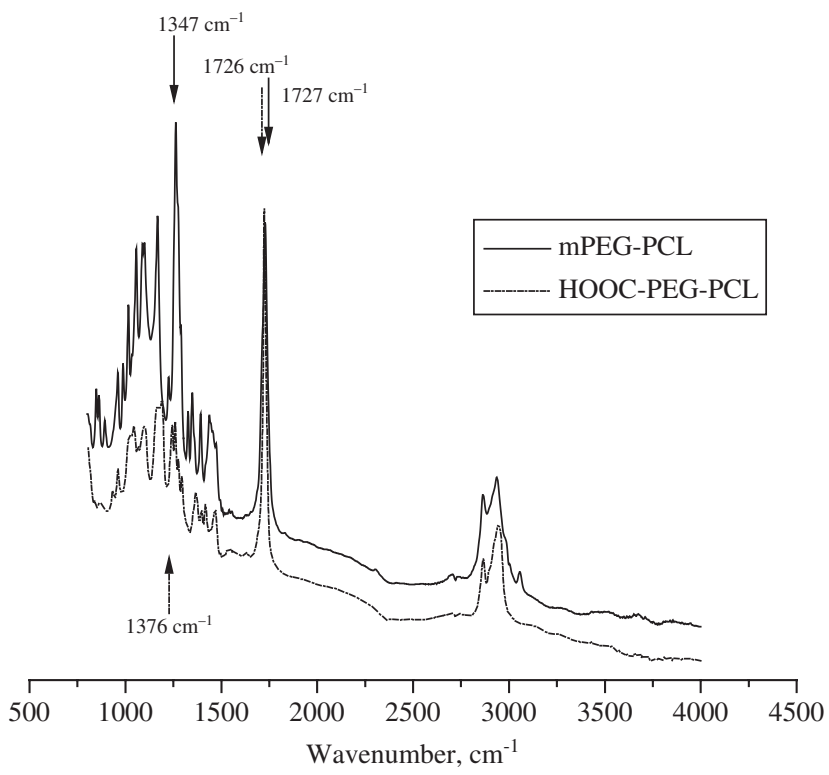

Figure 2: FTIR of HOOC-PEG-PCL and mPEG-PCL.

Different stirring rates as well as combinations of different stirring rates were tested in order to achieve nanoparticles with a narrow size distribution. Best results were achieved by a sequence of strong stirring rate $(750 \mathrm{rpm}$, $1 \mathrm{~h}$ ) followed by gentle stirring (350 rpm, $3 \mathrm{~h}$; Figure 3A). Using a continuously strong or continuously gentle stirring rate, only an irregular size distribution of the hydrodynamic diameter could be observed. Obviously, a strong or ongoing gentle stirring rate hamper the compounds to form uniform particles. In case of the gentle stirring rate, the copolymer formed huge particles of irregular size distribution. The hydrodynamic diameter of the particles was reproducible found to be in the range of $100-200 \mathrm{~nm}$ which was consistent with the characterization of the hydrodynamic diameter with NTA resulting in a diameter of 130-150 nm.

Next the time of sonication was modified (Figure 3B). Ultra-sonication is necessary to get small particles and break larger agglomerates. Using an ultra-sonication time of $120 \mathrm{~s}$ and $75 \mathrm{~s}$ a spacious size distribution of the hydrodynamic diameter was observed (Figure 3B). The optimal duration of ultra-sonication was at $90 \mathrm{~s}$. Leading to a small size distribution indicated by a defined peak (see arrow, Figure 3B) at 170-200 nm. In contrast, sonification enduring $75 \mathrm{~s}$ or $120 \mathrm{~s}$ revealed NPs with a broader size distribution. Thus, a sonication time of $90 \mathrm{~s}$ was used for all subsequent experiments. A possible reason for the broader size distribution observed after sonication for $75 \mathrm{~s}$ could be, that the sample is still containing some agglomerates which did not break during short sonication. 

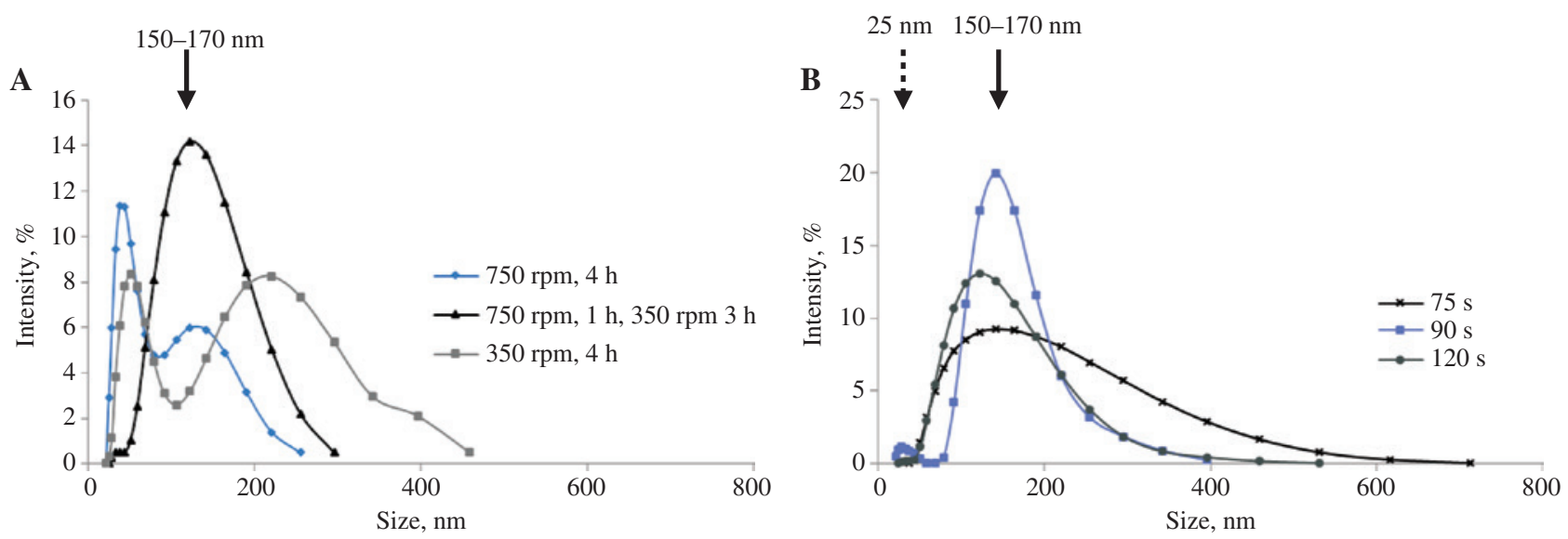

Figure 3: Hydrodynamic diameter of the particles produced with using different stirring rates $(A)$ and variations of ultra-sonication rates (B) characterized by DLS. There is a small peak at $25 \mathrm{~nm}$ and the maximum at $150-170 \mathrm{~nm}$.

In order to remove free copolymer that has not been incorporated into nanoparticles, overnight dialysis was performed. After dialysis with $3.5 \mathrm{kDa}$ and $6 \mathrm{kDa}$ DLS analysis revealed the absence of the peak of the hydrodynamic diameter at approximately $25 \mathrm{~nm}$, which was present in Figure $3 \mathrm{~A}$ and $\mathrm{B}$ before dialysis. Possibly this could be that $25 \mathrm{~nm}$ peak corresponds to monomers remaining in the solution after particle formation. The monomers are able to pass the dialysis membrane at a cut-off of $3.5 \mathrm{kDa}$ (Figure 4).

\section{Characterizing the particles}

The morphology of the particles was characterized by TEM. In Figure 5 spherical shaped particles are seen. The small size of $3 \mathrm{~nm}$ of the particles could be explained by the drying of the sample before TEM analysis. This results

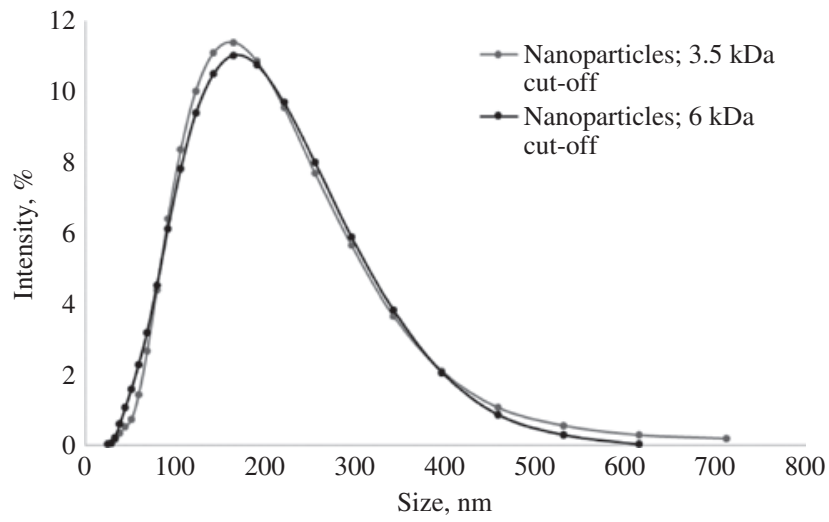

Figure 4: Different cut-offs during the dialysis after $350 \mathrm{rpm} 1 \mathrm{~h}$ and $750 \mathrm{rpm} 2 \mathrm{~h}$ and an ultra-sonication for $90 \mathrm{~s}$.

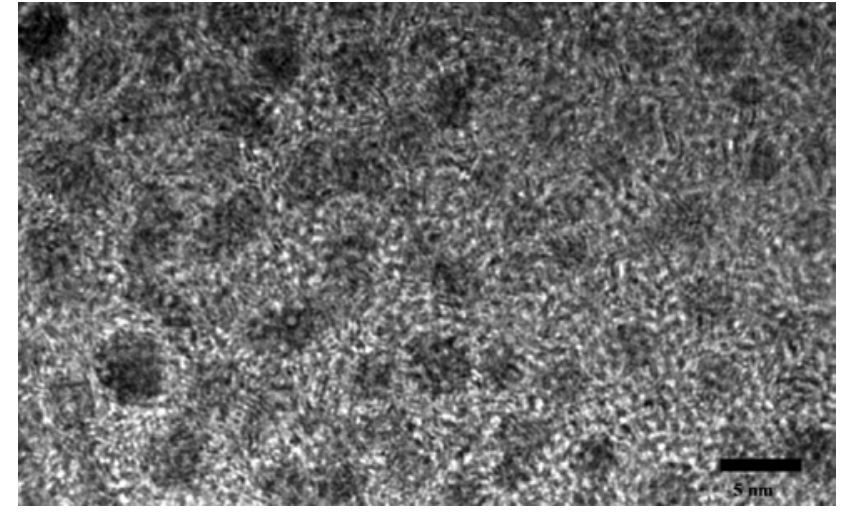

Figure 5: TEM analysis of the nanoparticles.

in a shrinking of the PEG shell of the particles. This observation has already been described in literature [28-30].

After determining the morphology of the particles their concentration was characterized. Nanoparticles' concentration was checked by lyophilisation and was reproducible at $2.8 \mathrm{mg} / \mathrm{mL}$.

After determining the morphology and concentration of the particles their surface charge was characterized from $\zeta$-potential measurements. Figure 6 shows the $\zeta$-potential of the nanoparticles.

NPs without drug loading and functionalization have a $\zeta$-potential at $-21 \mathrm{mV}$. Drug loaded NPs have a higher positive $\zeta$-potential of $-18 \mathrm{mV}$. This observation is in accordance with the literature [31]. NPs functionalized with aptamers without drug loading have a higher negative surface charge (between $-30 \mathrm{mV}$ and $-32 \mathrm{mV}$ ) than NPs without modification and drug loading. This is an anticipated result because aptamers are negatively charged and further confirms the successful modification 


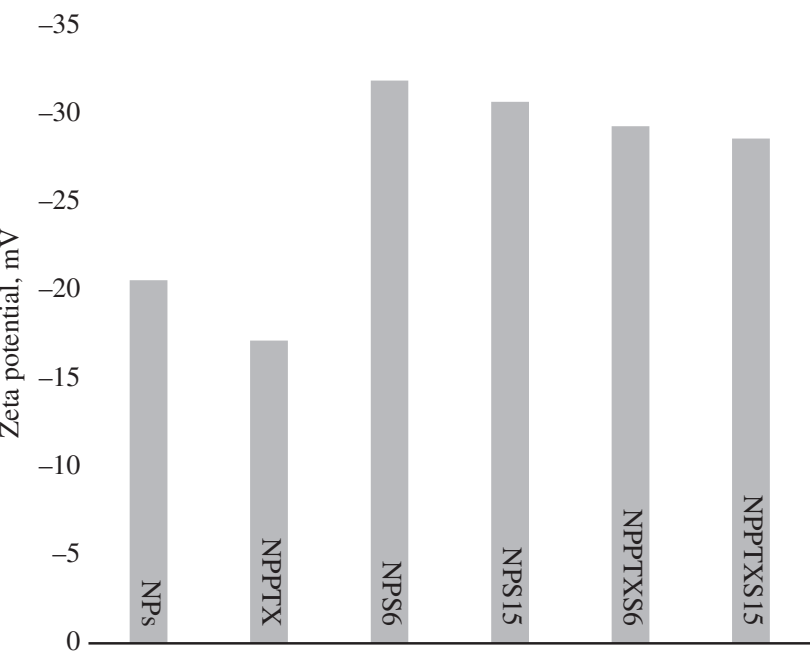

Figure 6: Zeta potential of the nanoparticles. NPs, nanoparticles; NPPTX, nanoparticles loaded with PTX; NPS6, nanoparticles functionalized with S6 aptamer; NPS15, nanoparticles functionalized with S15 aptamer; NPPTXS6, nanoparticles loaded with PTX and functionalized with S6 aptamer; NPPTXS15, nanoparticles loaded with PTX and functionalized with S15 aptamer.

of NPs with aptamers. NPs functionalized with the S6 aptamer generally have a lower $\zeta$-potential $(-32 \mathrm{mV}$ and $-30 \mathrm{mV}$ with PTX). A possible reason could be a different folding of the two aptamers resulting in differences of the charge distribution on the NP surface.

\section{Functionalization}

For a targeted drug delivery system, the drug carrier is modified with a targeting ligand. Aptamers are able to detect cell-specific receptors and molecules so in this study S15 and S6 aptamers were used which are directed against the lung cancer cell line A549. Aptamers were coupled via an EDC-coupling on the nanoparticles' surface. To remove the uncoupled aptamers, excessive buffer exchange was performed using centrifugal concentrators (100 kDa MWCO). Figure 7 shows the results of the functionalization with both aptamers.

While some unbound aptamer was found in the first washing fraction, in the following washing fractions no aptamer was detected. For the purified conjugates, a band corresponding to the aptamer was found. This indicates that most of the aptamer was bound to the nanoparticles and that free aptamer was successfully removed.

Hydrodynamic diameter of aptamer-conjugated nanoparticles was determined via DLS analysis (Figure 8). Nanoparticles with aptamer have a bigger size distribution of the hydrodynamic diameter than particles without aptamer. Here one has to consider that the modification of the nanoparticles with aptamers influences the hydrodynamic size of the particle via two contrary mechanisms: First, the size of the particle increases due to the presence of relatively large aptamers. On the other hand, aptamers are ssDNAs/RNAs oligonucleotides exhibiting a negative
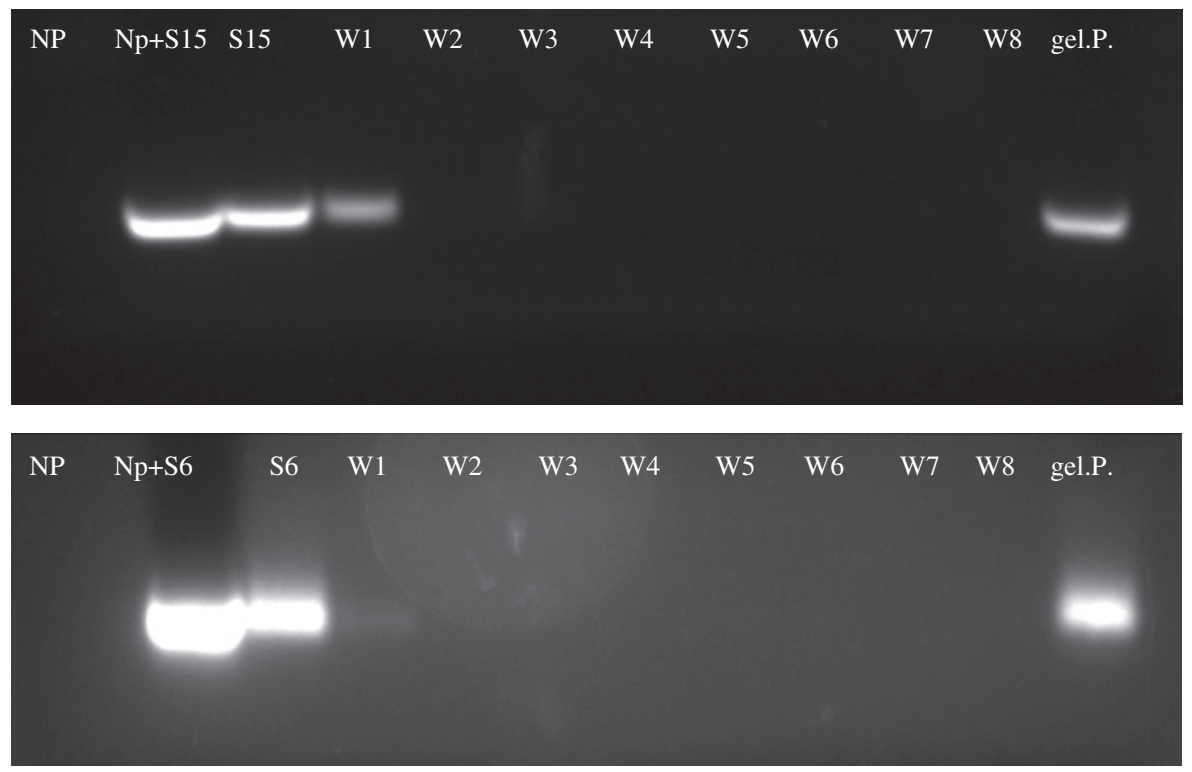

Figure 7: Top: Agarosegel of PEG-PCL-S15. NP, nanoparticles before conjugation; S15, aptamer directed against lung cancer cells; W1-W8, washing fractions, cut-off:100 kDa; gel.P., purified conjugate. Bottom: Agarosegel of PEG-PCL-S6. NP, nanoparticles before conjugtion; S6, aptamer directed against lung cancer cells; W1-W8, washing fractions, cut-off:100 kDa; gel.P., purified conjugate. 


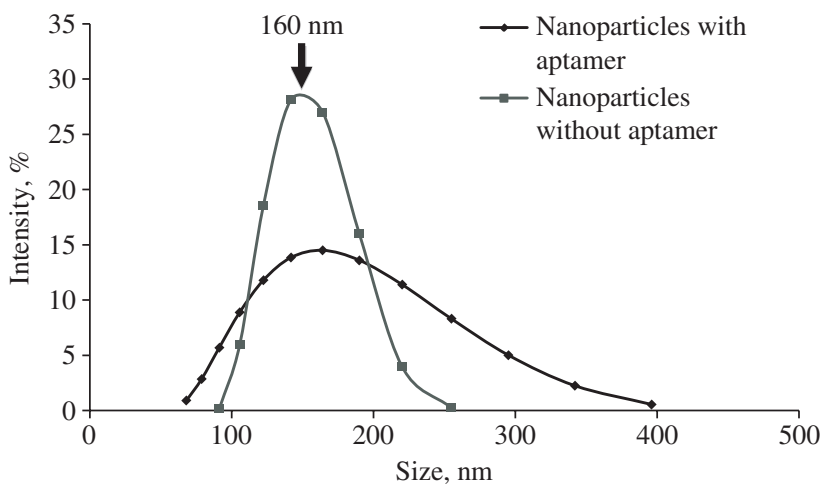

Figure 8: Nanoparticles with and without aptamer; characterized by DLS.

charge. The negative charge of the aptamer-modified particles results in a stronger attraction of the hydration shell and thus in smaller hydrodynamic diameters. Moreover, the hydrodynamic diameter may also be affected by the folding of the aptamer depending on the aptamer density: At low aptamer densities, the aptamer may be more flexible than at higher aptamer densities for which the electrostatic repulsion of negatively charged aptamers results in a straightening of the aptamers [32]. In summary our results suggest successful conjugation with aptamerconjugated nanoparticles with a hydrodynamic diameter between $100 \mathrm{~nm}$ and $300 \mathrm{~nm}$ and a maximum at $160 \mathrm{~nm}$.

\section{Encapsulation of PTX}

PTX was encapsulated during the dissolution of the two compounds of the particles in DCM [16, 19]. After dissolving all three compounds, the solution was dropwise added to sodium cholate hydrate solution. When an amount of $200 \mu \mathrm{g} / \mathrm{mL}$ PTX was used during particle formation, the encapsulation efficiency was nearly $44 \%$ and $43.89 \mu \mathrm{g} /$ $\mathrm{mL}$ of PTX were encapsulated. So the maximum amount of PTX which could reach the cells is about $7.3 \mathrm{ng}$ per cell. Particles loaded with PTX were used for further investigation of cytotoxicity.

\section{Incubation of cells with drug loaded and functionalized NPs}

Nanoparticles $(2.8 \mathrm{mg} / \mathrm{mL})$ loaded with $200 \mu \mathrm{g} / \mathrm{mL}$ PTX [16] were incubated with A549 cells.

We have recently used aptamer S15-functionalized quantum dots for specific staining of A549 cells [33]. We also have compared the binding of S15 to A549 cells in comparison to a random oligonucleotide as a negative control. So we could demonstrate that S15 is specific for A549 cells [18]. According to propidium iodide staining in Figure 9A, A549 cells incubated with PEG-PCL-S15 (PTX) particles died Calcein-AM staining revealed absence of living cells under these conditions. In contrast, the control (HeLa cells, Figure 9C) showed no dead cells. Although a certain affinity of aptamer S15 has been described in the literature, the binding to HeLa was reported to be weak when compared to aptamer binding to A549 cells [25]. Therefore, our results demonstrate the higher specificity of the conjugates to A549 cells.

The sample with S6 aptamer (PEG-PCL-S6) showed no dead cells for both, A549 cells and HeLa cells. A possible reason could be that $\mathrm{S} 6$ is binding to different receptors of A549 cells that do not trigger the endocytosis. In another set of experiments, drug-loaded nanoparticles not functionalized with aptamers (PEG-PCL (PTX)) were used as a negative control resulting in no increase of dead cells (data not shown). A549 and HeLa did not die as well after incubation with PEG-PCL-S15 and PEG-PCL-S6 (data not shown). Therefore, the specific targeting and cellular uptake was demonstrated for S15-modified particles, whereas S6-conjugated particles failed in penetration of the cell membrane.

Cells incubated with unloaded NPs or with NPs not functionalized with an aptamer did not die, meaning that the composition of NPs is not toxic for the cells (Figure 9E). Cells incubated with pure taxol died (up to $50 \%$ ) after the incubation time of $24 \mathrm{~h}$ (data not shown).

\section{Conclusion and further experiments}

The aim of the study was to develop a model system for targeted drug delivery and its optimization. Nanoparticles could be produced successfully and the production was optimized. Furthermore their hydrodynamic diameter was characterized and also their morphology. PTX was encapsulated successfully with an efficiency of $44 \%$. Finally, functionalization with two different targeting ligands was confirmed.

To investigate the cytotoxicity and specificity of the developed drug delivery system A549 and HeLa cells were incubated with drug-loaded and aptamer-functionalized NPs, drug loaded NPs and only NPs (PEG-PCL-S15 (PTX), PEG-PCL-S15, PEG-PCL-S6 (PTX), and PEG-PCL-S6). The results demonstrated specific delivery and cellular uptake into A549 cells for S15-modified NPs. S6-modified particles were not able to deliver the incorporated drug into A549 

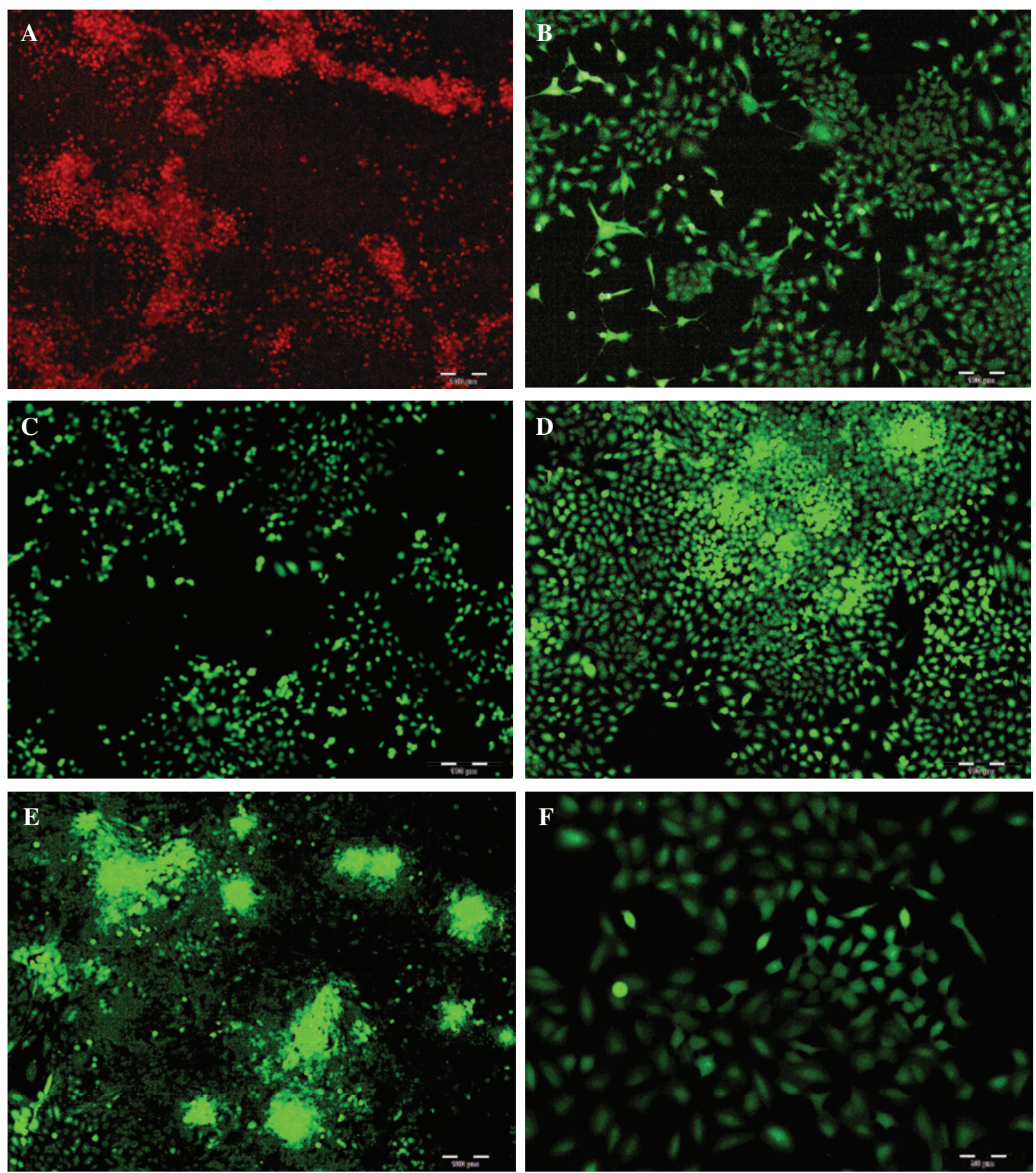

Figure 9: Staining with Calcein-AM/propidium iodide A: A549 cells were incubated with PEG-PCL-S15 (PTX) for 24 h; B: A549 cells were incubated with PEG-PCL-S6 (PTX) for 24 h; C: HeLa cells were incubated with PEG-PCL-S15 (PTX) for 24 h; D: HeLa cells were incubated with PEG-PCL-S6 (PTX) for 24 h. E: A549 cells were incubated with PEG-PCL particles. F: HeLa cells were incubated with PEG-PCL particles.

cells most likely because the aptamers molecular target does not facilitate endocytosis. In future work we will further optimize aptamer density and investigate the cytotoxicity of different drugs and drug concentrations. We also will investigate interactions between NPs and cells by incorporation of fluorescent dyes to the NPs and analysis of cellular binding and uptake of these NPs via confocal microscopy. Finally cytotoxicity tests with other cancer cells and healthy cells (esp. liver cells) will be performed.

\section{Materials and methods}

\section{Materials}

Monomethoxy-polyethylene glycol (mPEG, MW=5000 Da), R-carboxyl polyethylene glycol (HOOC-PEG, MW=114.14 Da), Coumarin-6, $\mathrm{N}$-(3-dimethylaminopropyl)- $\mathrm{N}^{\prime}$-ethylcarbodiimide hydrochloride (EDC), sodium cholate hydrate, boric acid (99\%), paclitaxel (PTX) and Dulbecco's Modified Eagle's Medium (DMEM) were purchased 
from Sigma Aldrich (Germany). A549 cell line (lung cancer) and HeLa (human cervix cancer) cells were acquired from 'Deutsche Sammlung von Mikroorganismen und Zellkulturen GmbH (DMSZ). Supplements for the cell culture medium DMEM and fetal bovine serum (FKS) were purchased from PAA Laboratories $\mathrm{GmbH}$, and penicillin/streptomycin (P/S) was purchased from Biochrom GmbH, Germany.

The S15 aptamer has the following sequence and a $5^{\prime}$ aminoC6 linker: GCTATCTTATGG AAATTTCGTGTAGGGTTTGGTGTGGCGGGGCTA. The S6 aptamer has the following sequence and a $5^{\prime}$ amino-C6 linker: GTGGCCAGTCACTCAATTGGG TGTAGGGG TGGGGATTGTGGGTTG [25]. Both S15 and S6 aptamers were purchased from BioSpring Gesellschaft für Biotechnologie mbH, Frankfurt/Main.

Agarose was purchased from Carl Roth $\mathrm{GmbH}$, Karlsruhe, TAE-buffer for the agarose gel electrophoresis consists of 2-Amino2-(hydroxylmehtyl)-1,3-propandiole (TRIS-Base) which was also purchased from Sigma Aldrich GmbH (Germany), glacial acetic acid which was acquired from AppliChem GmbH, Darmstadt and EDTA (Ethylenediaminetetraacetate) which was purchased from Sigma Aldrich GmbH (Germany). Roti-Safe Gelstain was also purchased from Carl Roth GmbH, Karlsruhe.

\section{Synthesis and characterization of copolymers}

Methoxy polyethylene glycol (mPEG) was dehydrated by an azeotropic distillation in toluene $(30 \mathrm{~mL})$ and then dried by vacuum at $50{ }^{\circ} \mathrm{C}$. $\varepsilon$-Caprolactone was dried under vacuum for $24 \mathrm{~h} .5 \mathrm{~g}$ of the $\varepsilon$-Caprolactone, $5 \mathrm{~g}$ of the $\mathrm{mPEG}$ and the catalyst stannous octoate $(0.1 \%)$ are added into a $50 \mathrm{~mL}$ dried glass reactor and the ringopening copolymerization was carried out at $130{ }^{\circ} \mathrm{C}$ for $24 \mathrm{~h}$ under reduced pressure (stirred slowly). The reaction was terminated by cooling in water [19]. HOOC-PEG-PCL was synthesized identically. The success of the copolymerization was characterized by ${ }^{1} \mathrm{H}$ NMR (Varian VNMRS 400; $400 \mathrm{MHz}, \mathrm{CDCl}_{3}$ ) and FTIR (IFS 66 BRUKER, equipped with an internal reflection element $45^{\circ} \mathrm{ZnSe}$ crystal and a deuterated triglycine sulfate (DTGS) detector).

\section{Preparation of nanoparticles}

The nanoparticles (NPs) were synthesized by a solvent evaporation method. $28 \mathrm{mg}$ of mPEG-PCL and $3 \mathrm{mg}$ HOOC-PEG-PCL were dissolved in $1 \mathrm{~mL}$ of dichloromethane (DCM) and then dropwise added to $5 \mathrm{~mL}$ of a $0.6 \%$ sodium cholate hydrate solution. For the encapsulation of PTX, different amounts of PTX $(200 \mu \mathrm{g} / \mathrm{mL})$ were added to the mPEG-PCL-DCM solution.

The solution was stirred for $1 \mathrm{~h}$ at $750 \mathrm{rpm}$ and for $2 \mathrm{~h}$ at $350 \mathrm{rpm}$ and then pulse sonicated for $90 \mathrm{~s} 200 \mathrm{~W}$. Stirring rate and ultrasonication rate were varied in case of optimization of nanoparticles' preparation. Then the mixture was applied to a rotary evaporator to remove DCM. To remove unreacted monomers and PTX which was not encapsulated, a dialysis was performed with cut-offs of $3.5 \mathrm{kDa}$ and $6 \mathrm{kDa}$.

For the functionalization with the S15 and S6 aptamers the carboxylated PEG of the nanoparticle was activated by EDC. Therefore, the aptamer $(177 \mu \mathrm{M}, 16 \mu \mathrm{L})$ was added to NP solution $(2.8 \mathrm{mg} / \mathrm{mL}$, $24.4 \mu \mathrm{L}$ ) in sodium borate buffer (SBB; $10 \mathrm{mM}, \mathrm{pH} 7.4)$. After mixing the solution on a rotary mixer (labtech) for $5 \mathrm{~min}, 6.5 \mu \mathrm{L}$ of a $10 \mathrm{mg} / \mathrm{mL}$ EDC-solution were added and then incubated for $3 \mathrm{~h}$ at room temperature on the rotary mixer. Afterwards the conjugates were purified using centrifugal concentrators (Vivaspin ${ }^{\circledR}, 100 \mathrm{kDa}$ cut-off). This process was used to remove unreacted aptamers; success was checked by agarose gel electrophoresis $(1.2 \%, 100 \mathrm{~V}$, stained with $5 \mu \mathrm{L} / 100 \mathrm{~mL}$ agarose gel Roti-Safe Gel-Stain).

\section{Characterization of NPs}

Particle size was determined by dynamic light scattering (DLS, Malvern Instruments Ltd., zetasizer Nano ZS, UK). The surface charge of the NPs was determined from $\zeta$-potential measurements (Malvern Instruments Ltd., zetasizer Nano ZS, UK). The exactly number of particles per mL was measured by nanotracking analysis (NTA, Malvern Instruments Ltd., zetasizer Nano ZS, UK). Morphology was characterized by transmission electron microscopy (TEM, FEI Tecnai G2 F20 TMP-TEM with a $200 \mathrm{kV} \mathrm{FEG} \mathrm{(field} \mathrm{emission} \mathrm{gun)).} \mathrm{Samples} \mathrm{were} \mathrm{pre-}$ pared by placing a droplet on a carbon coated mesh copper grid and then they were dried with air. For determination of the concentration of the particles a lyophilization (Eppendorf concentrator 5301) was done.

\section{Encapsulation efficiency of PTX in nanoparticles}

The encapsulation efficiency was determined by high performance liquid chromatography (HPLC, VWR Hitachi Chromaster). The mobile phase consisted of acetonitrile/water (50:50 v/v). Column (Kinetex $\mathrm{C} 18,2.6 \mu \mathrm{m}, 100 \mathrm{~A}, 100 \times 4.6 \mathrm{~mm}$ ) temperature was at $25^{\circ} \mathrm{C}$ and the flow rate was set at $1 \mathrm{~mL} / \mathrm{min}$; detection wavelength was $225 \mathrm{~nm}$. Sample solution was injected at a volume of $10 \mu \mathrm{L}$. The calibration was done with standard solutions of PTX $(5-1000 \mu \mathrm{g} / \mathrm{mL})$ dissolved in water. Analyzing the encapsulation efficiency of the PTX was performed using the calibration data with the Agilent series OpenLAB Control Panel software. To calculate the encapsulation efficiency following formula was used [16]:

$$
\text { encapsulation efficiency [\%] }=\frac{\text { amount of PTX in nanoparticles }}{\text { initial amount of PTX }} \times 100
$$

\section{Optimizing of NP preparation}

The NP preparation was optimized with regard to size distribution. Therefore, stirring rate and ultra-sonication rate were varied as well as the molar ratio of the compounds. Therefore, the amount of HOOCPEG-PCL was changed. In this study we tested the influence of $2 \mathrm{mg}$ HOOC-PEG-PCL, $3 \mathrm{mg}$ and $4 \mathrm{mg}$ on the hydrodynamic diameter of the particles. The stirring rate was varied between $350 \mathrm{rpm}$ and $750 \mathrm{rpm}$ for variable times. The ultra-sonication time was varied between $75 \mathrm{~s}$ and $120 \mathrm{~s}$.

\section{Cytotoxicity tests}

A549 cells were seeded at a density of $1 \times 10^{3}$ cells per $\mathrm{cm}^{2}$ in a 96 well plate and cultivated to confluency. Consequently, they were incubated with drug-loaded and aptamer-functionalized NPs. As negative 
controls drug-loaded NPs without aptamer modification and NPs without any drug loading and functionalization were used. After $24 \mathrm{~h}$ incubation cells were stained with calcein-acetoxymethyl (AM)/ propidium iodide. This staining provides information about the ratio of dead and living cells. The non-fluorescent ester calcein-AM penetrates the cell membrane and is split by metabolic active cells. Calcein forms green fluorescent chelate complexes with iron ions thereby only staining living cells. In contrast, propidium iodide cannot penetrate membranes of living cells and only penetrates membranes of dead cells.

Acknowledgments: Julia Modrejewski would like to acknowledge Hannover School for Biomolecular Drug Research (HSBDR) for financial support.

\section{References}

1. Bertrand N, Leroux, J-C. The journey of a drug-carrier in the body: an anatomo-physiological perspective. J Control Release. 2012;161:152-63.

2. Priya James H, John R, Alex A, Anoop KR. Smart polymers for the controlled delivery of drugs-a concise overview. Acta Pharm Sin B. $2014 ; 4: 120-7$.

3. Coelho, JF, Ferreira, PC, Alves, P, Cordeiro, R, Fonseca, AC, Góis, JR, et al. Drug delivery systems: advanced technologies potentially applicable in personalized treatments. EPMA. 2010;1:164-209.

4. Ruggiero C, Pastorino L, Herrera OL. Nanotechnology based targeted drug delivery. Conf Proc IEEE Eng Med Biol Soc. 2010;2010:3731-2.

5. Moorthi C, Manavalan R, Kathiresan K. Nanotherapeutics to overcome conventional cancer chemotherapy limitations. J Pharm Pharmaceut Sci. 2011;14:67-77.

6. Jong WH, de; Borm, PJA. Drug Delivery and nanoparticles: applications and hazards. Int J Nanomedicine. 2008;3:133-49.

7. Trafton A. Tumors targeted using tiny gold particles. MIT Tech Talk. 2009;53:4.

8. Zheng XL. Kan B, Gou ML, Fu SZ, Zhang J, Men K, et al. Preparation of MPEG-PLA nanoparticle for honokiol delivery in vitro. Int J Pharm. 2010;386:262-7.

9. Singh R, Lillard JW. Nanoparticle-based targeted drug delivery. Exp Mol Pathol. 2009;86:215-23.

10. Farokhzad, OC, Langer, R. Impact of nanotechnology on drug delivery. ACS Nano. 2009;3:16-20.

11. Papazoglou E, Parthasarathy A. BioNanotechnology. San Rafael, CA: Morgan \& Claypool Publishers; 2007.

12. Gullotti E, Yeo Y. Extracellularly activated nanocarriers: a new paradigm of tumor targeted drug delivery. Mol Pharm. 2009;6:1041-51.

13. Sudimack J, Lee RJ. Targeted drug delivery via the folate receptor. Adv Drug Deliv Rev. 2000;41:147-62.

14. Patri AK, Kukowska-Latallo JF, Baker JR. Targeted drug delivery with dendrimers: comparison of the release kinetics of covalently conjugated drug and non-covalent drug inclusion complex. Adv Drug Deliv Rev. 2005;57:2203-14.

15. Jain KK, editor. Drug delivery systems. Totowa, NJ: Humana Press; 2008.
16. Danhier, F, Lecouturier, N, Vroman, B, Jérôme, C, MarchandBrynaert, J, Feron, O, et al. Paclitaxel-loaded PEGylated PLGAbased nanoparticles: in vitro and in vivo evaluation. J Control Release. 2009;133:11-17.

17. Li XY, Kong XY, Shi S, Wang XH, Guo G, Luo F, et al. Physical, mechanical and biological properties of poly( $\varepsilon$-caprolactone)poly(ethylene glycol)-poly( $\varepsilon$-caprolactone) (CEC)/chitosan composite film. Carbohydr Polymer. 2010;82:904-12.

18. Ulusoy M, Jonczyk R, Walter J-G, Springer S, Lavrentieva A Stahl F, et al. Aqueous Synthesis of PEGylated Quantum Dots with Increased Colloidal Stability and Reduced Cytotoxicity. Bioconjug Chem. 2015a;27:414-26.

19. Gao H, Qian J, Cao, S, Yang Z, Pang Z, Pan, S, et al. Precise glioma targeting of and penetration by aptamer and peptide dual-functioned nanoparticles. Biomaterials. 2012;33: 5115-23.

20. Bharadwaj, R, Yu, H. The spindle checkpoint, aneuploidy, and cancer. Oncogene. 2004;23:2016-27.

21. Cho, K, Wang, X, Nie, S, Chen, ZG, Shin, DM. Therapeutic nanoparticles for drug delivery in cancer. Clin Cancer Res. 2008;14:1310-6.

22. Friedman AD, Claypool SE, Liu R. The smart targeting of nanoparticles. Curr Pharm Des. 2013;19:6315-29.

23. Lönne, M, Bolten, S, Lavrentieva A, Stahl F, Scheper T, Walter J-G. Development of an aptamer-based affinity purification method for vascular endothelial growth factor. Biotechnol Rep. 2015;8:16-23.

24. Wu X, Chen J, Wu M, Zhao JX. Aptamers: active targeting ligands for cancer diagnosis and therapy. Theranostics. 2015;5:322-44.

25. Zhao Z, Xu L, Shi X, Tan W, Fang X, Shangguan D. Recognition of subtype non-small cell lung cancer by DNA aptamers selected from living cells. Analyst. 2009;134:1808-14.

26. Walter J-G, Heilkenbrinker A, Austerjost J, Timur S, Stahl F, Schepe T. Aptasensors for small molecule detection. Z Naturforsch. 2012;67b:976-86.

27. Tuerk C, Gold L. Systematic evolution of ligands by exponential enrichment: RNA ligands to bacteriophage T4 DNA polymerase. Science. 1990;249:505-20.

28. Yoshio W, Atsushi M, editors. Morphology control of materials and nanoparticles: advanced materials processing and characterization. Berlin: Springer-Verlag; 2004.

29. Nanthakasri, W. Biodegradable blend nanoparticles of amphiphilic diblock copolymers prepared by nano-precipitation method. J Biomater Nanobiotechnol. 2011;2:561-6.

30. Baimark Y, Srisuwan Y. Biodegradable nanoparticles of methoxy poly (ethlene glycol)- $b$-poly(D,L-lactide)/methoxy poly (ethylene glycol)- $b$-poly( $\varepsilon$-cparolactone) blends for drug delivery. Nanoscale Res Lett. 2012;7:271.

31. Xin H, Chen L, Gu J, Ren X, Wei Z, Luo J, et al. Enhanced anti-glioblastoma efficacy by PTX-loaded PEGylated poly( $\varepsilon$-caprolactone) nanoparticles: In vitro and in vivo evaluation. Int J Pharm. 2010;402:238-47.

32. Walter JG, Petersen S, Stahl F, Scheper T, Barcikowski S. Laser ablation-based one-step generation and bio-functionalization of gold nanoparticles conjugated with aptamers. J Nanobiotechnology. 2010;8:21.

33. Ulusoy M, Walter J-G, Lavrentieva A, Kretschmer I, Sandiford L, Le Marois A, et al. One-pot aqueous synthesis of highly strained $\mathrm{CdTe} / \mathrm{CdS} / \mathrm{ZnS}$ nanocrystals and their interactions with cells. RSC Adv. 2015b;5:7485-94. 